\title{
3 Research Square

\section{Displacement in root apex and changes in incisor inclination affect alveolar bone remodeling in adult bimaxillary protrusion patients: a retrospective study}

\author{
Huimin Mao \\ Nanjing Stomatological Hospital \\ Andi Yang \\ Nanjing Stomatological Hospital \\ Yue Pan \\ Nanjing Stomatological Hospital \\ Houxuan Li \\ Nanjing Stomatological Hospital \\ Lang LEI ( $\sim$ leilangdental@163.com ) \\ Nanjing Stomatological Hospital https://orcid.org/0000-0003-2892-040X
}

\section{Research}

Keywords: bimaxillary protrusion, alveolar bone remodeling, orthodontic tooth movement, periodontal

Posted Date: April 16th, 2020

DOI: https://doi.org/10.21203/rs.3.rs-22651/v1

License: (c) (i) This work is licensed under a Creative Commons Attribution 4.0 International License. Read Full License

Version of Record: A version of this preprint was published on November 20th, 2020. See the published version at https://doi.org/10.1186/s13005-020-00242-2. 


\section{Abstract}

Background: Periodontal health is of great concern for periodontists and orthodontists in the interdisciplinary management of patients with bimaxillary protrusion. The aim of present study is to investigate changes in the alveolar bone in the maxillary incisor region and to explore its relationship with displacement of root apex as well as changes in the inclination of maxillary incisors during incisor retraction.

Methods: Samples in this retrospective study consisted of 38 patients with bimaxillary protrusion. Conebeam computed tomography (CBCT) images was taken before(T0) and after ( $\mathrm{T} 1)$ treatment. Alveolar bone thickness $(A B T)$, height $(A B H)$ and area $(A B A)$ were utilized to evaluate changes in the alveolar bone, while incisor inclination and apex displacement were used to assess changes in the position of maxillary central and lateral incisors. Correlations between alveolar bone remodeling and apex displacement as well as changes in the inclination were investigated.

Results: The labial ABT of central and lateral incisors at the mid-root third was increased. In contrast, the palatal ABT at crestal, mid-root and apical third level were consistently decreased. ABH was not altered on the labial side, while significantly decreased on the palatal side. ABA was not significantly increased on the labial side, but significantly decreased on the palatal side, leading to a significantly reduced total ABA. Orthodontic treatment significantly reduced inclination of upper incisors. Changes in the amount (T1-T0) of ABA was remarkably correlated with apex displacement and changes of inclination (T1-T0); in addition, using the multivariate linear regression analysis, changes of ABA on the palatal side (T1-T0) can be described by following equation: Changes of palatal $\mathrm{ABA}(\mathrm{T} 1-\mathrm{T} 0)=-3.258-0.139 \times$ changes of inclination (T1-T0) + 2.533×apex displacement (T1-T0).

Conclusions: Retraction of incisors in bimaxillary protrusion patients may compromise periodontal bone support on the palatal side. An equation that incorporated the displacement of root apex and change in the incisor inclination may enable periodontist-orthodontist interdisciplinary coordination in assessing treatment risks and developing an individualized treatment plan for adult patients with bimaxillary protrusion.

\section{Background}

Bimaxillary protrusion, a well-recognized malocclusion in Eastern Asian communities, is characterized by proclined incisors as well as protruded lips ${ }^{[1]}$. Negative perception of protrusive dentitions and lips is one major concern for patients to seek orthodontic care. Four-premolar extraction, maximal anchorage and incisor retraction are often included in the treatment to achieve optimal improvement in the lip morphology ${ }^{[2]}$. Although dynamic remodeling of alveolar bone might ensue orthodontic tooth movement $(\mathrm{OTM})^{[3-5]}$, the large retraction of incisors poses a great challenge to the periodontal health in patients with bimaxillary protrusion. Delicate treatment planning and risk assessment may help reduce the periodontal sequelae in patients with bimaxillary protrsution. 
Despite the persistent controversy regarding the alveolar bone remodeling pattern during OTM, i.e. " through the bone" or "with the bone" theory ${ }^{[6]}$, the 2014 American Association of Periodontal

Regeneration World Workshop systematic review reports that the direction of the tooth movement and the bucco-lingual thickness of the gingiva play important roles in soft tissue alterations during orthodontic treatment ${ }^{[7]}$. Therefore, to reduce the periodontal risk in patients with bimaxillary protrusion, an interdisciplinary approach involving the orthodontists and periodontists in the decision-making process would definitely reduce periodontal sequelae and improve orthodontic treatment outcome ${ }^{[8]}$. Anatomic features of local alveolar process, periodontal remodeling potential and orthodontic tooth movement pattern should be included in determining overall treatment plan and specific force system.

Cone-beam computed tomography (СBCT) images are being widely used in orthodontics to assess 3dimensional(3D) spatial relationship in the diagnosis of impacted teeth ${ }^{[9]}$. In addition, 3D imaging allows for accurate detection of anatomic features of alveolus, which is superior to lateral cephalometric, intraoral periapical or panoramic radiographs ${ }^{[10]}$. Information on the alveolar bone surrounding incisors is of vital importance to determine the boundary of orthodontic tooth movement.

In addition to assessment of periodontal tissue condition and remodeling potential, delicate orthodontic treatment plan and precise tooth movement control should also be guaranteed to minimize periodontal risks. Upon application of orthodontic forces on the periodontal ligament, biological responses in the molecular and cellular level will lead to several types of OTM, including uncontrolled tipping, controlled tipping and bodily movement ${ }^{[11]}$. Such differed types of OTM may lead to varied displacement of the apex and incisor inclination although retraction of incisal edge is similar.

Orthodontic tooth movement is limited by the anatomic dentoalveolar boundaries that are set by the cortical plates of the alveolus at the level of the incisor apices ${ }^{[4]}$. Digital setup by available software, such as OrthoCAD (Align Technology, San Jose, Calif), SureSmile (Orametrix, Richardson, Tex), and Orchestrate (Orchestrate, Rialto, Calif), has been widely utilized to mimic tooth movement and predict final position of the dentition rather accurately ${ }^{[12]}$. However, without taking the alveolar anatomy and its remodeling capacity into consideration, digital setup-aided orthodontics as well as traditional orthodontics may possibly put the dentition out of the boundary, leading to instability, root resorption and alveolar bone loss $^{[7]}$. Present data regarding the correlation between incisor retraction and alveolar bone remodeling is rather limited. To facilitate dentoalveolar bone risk assessment in the inter-disciplinary management of bimaxillary protrusion, we aimed to investigate dynamic changes in the alveolar bone in the incisor region and to explore its relationship with displacement of root apex as well as changes in the inclination of maxillary incisors.

\section{Methods}

\section{Materials}


This retrospective study was approved by the Institutional Ethics Committee. The IRB number is 2019NL064(KS). The study included data from 38 patients (31 females and 7 males), retrieved from the archive of medical school of university, from 2014 to 2019. The mean preoperative age was 19.52 years with an overall range of 15-33 years. All patients were treated with straight wire appliances (SWA) with an MBT prescription after extraction of four first premolars. Extraoral arch or temporary anchorage devices (TADs) was adopted to achieve maximal anchorage, and sliding mechanics with en masse retraction were utilized in all the subjects in the $0.022 " \times 0.028$ " slot system. Treatment time was $26 \pm 4$ month and the cephalometric data was presented in Table 1.

Table 1

The cephalometric data before and after orthodontic treatment (Mean \pm $\mathrm{SD})$.

\begin{tabular}{|c|c|c|c|}
\hline Parameter & TO & $\mathrm{T} 1$ & P Value \\
\hline $\operatorname{SNA}\left({ }^{\circ}\right)$ & $81.7 \pm 3.1$ & $81.2 \pm 2.2$ & $0.4^{\mathrm{W}}$ \\
\hline $\operatorname{SNB}\left({ }^{\circ}\right)$ & $75.7 \pm 3.1$ & $75.6 \pm 2.4$ & $0.8^{\top}$ \\
\hline $\operatorname{ANB}\left({ }^{\circ}\right)$ & $6.0 \pm 1.3$ & $5.6 \pm 1.2$ & $0.1^{\top}$ \\
\hline $\mathrm{U} 1-\mathrm{SN}\left({ }^{\circ}\right)$ & $108.8 \pm 6.7$ & $99.2 \pm 6.5$ & $\star \star \star * T$ \\
\hline U1-NA( $\left(^{\circ}\right)$ & $26.4 \pm 5.7$ & $19.0 \pm 6.9$ & $\star \star \star * T$ \\
\hline $\mathrm{U} 1-\mathrm{NA}(\mathrm{mm})$ & $2.4 \pm 0.8$ & $1.6 \pm 0.7$ & $\star \star \star * T$ \\
\hline MP-SN( $\left(^{\circ}\right)$ & $39.3 \pm 5.7$ & $38.8 \pm 5.2$ & $0.6^{\top}$ \\
\hline $\mathrm{MP}-\mathrm{FH}\left({ }^{\circ}\right)$ & $30.0 \pm 5.7$ & $30.3 \pm 4.8$ & $0.4^{\mathrm{W}}$ \\
\hline L1-MP( $\left.{ }^{\circ}\right)$ & $98.5 \pm 6.4$ & $92.1 \pm 7.5$ & $\star \star \star * T$ \\
\hline L1-NB $\left({ }^{\circ}\right)$ & $34.1 \pm 5.9$ & $27.3 \pm 6.6$ & $\star \star * * T$ \\
\hline L1-NB(mm) & $4.7 \pm 1.2$ & $3.1 \pm 0.9$ & $* \star * T$ \\
\hline U1-L1( $\left(^{\circ}\right)$ & $113 \pm 6.704$ & $129.3 \pm 8.7$ & $* * * T$ \\
\hline \multicolumn{4}{|c|}{ T, paired t-test; W, Wilcoxon test. ${ }^{*}, \mathrm{P}<0.05 ;{ }^{* *}, \mathrm{P}<0.01$; $* \star *, \mathrm{P}<0.00$} \\
\hline
\end{tabular}

Inclusion criteria for bimaxillary protrusion patients are:

(1) With complete CBCT data before(T0) and after treatment(T1)

(2) Class I canine and molar relationship; 
(3) Pretreatment interincisal angle less than $124^{\circ}$ with a crowding of less than $4 \mathrm{~mm}$ in the maxillary arch;

(4) Full permanent dentition anterior to the first molars;

(5) A minimum age of 15 in girls and 18 in boys to reduce effects of growth on dental structures.

Patients with previous orthodontic treatment, cleft lip palate, impacted anterior teeth, congenital tooth loss except third molars, systemic diseases and compromised periodontium were excluded.

\section{D Image Processing and Measurements}

All pretreatment and posttreatment CBCT were taken by the same machine. CBCT scans (NewTomVG, Italy) were taken before ( $\mathrm{T} 0$ ) and after treatment ( $\mathrm{T} 1)$. The following imaging acquisition parameters were used: $5 \mathrm{~mA}, 110 \mathrm{kV}$, and 3.6 seconds exposure time, which generated an isotropic voxel size of $0.3 \mathrm{~mm}$.

The examined subjects were positioned in the sagittal plane perpendicular to the floor, which was parallel to the Frankfort plane. All CBCT data were exported to digital imaging and communications in medicine (DICOM) format. The 3D images were reconstructed using NNT Viewer software (NewTomVG, Italy).

In NNT Viewer, the sagittal slices were cut through the center of apical foramen and parallel to the long axis of individual incisors. These images were imported into the software ImageJ (version 2.0, NIH, Bethesda, Md), within which the following variables were measured: (1) alveolar bone thickness( $A B T)$ on the buccal and palatal side at 3, 6 and $9 \mathrm{~mm}$ from the cementoenamel junction (CEJ), which is designated as crestal, mid-root and apical third; (2) labial and palatal alveolar bone area (ABA); (3) labial and palatal alveolar bone height $(A B H)$, defined as the distance from the CEJ to the alveolar ridge crest (Fig. 1A and B).

Tooth movement of individual maxillary incisor was determined by apex displacement and axis inclination alteration (modified from Kyoung-Won et. al.) ${ }^{[13]}$. The apical displacement was registered as the changes of the distance between the upper incisors' apical foramen and the palatal cortical bone, with apex moving towards buccal plates defined as positive and towards palatal plates as negative. The rationale of the reference mark is that the palatal cortical plate does not undergo structural changes and remain to be stable in adult patients ${ }^{[14]}$. Moreover, this reference mark is very close to the investigated area avoiding unwanted changes of remote structures and minimizing errors of measurement. Inclination of individual incisor was determined as the lower backward angle between the long axis and Frankfurt horizontal (FH) plane.

\section{Statistical Analyses}

All measurements were conducted by one trained examiner. To reduce the measurement error, we took the average value of three measurements whose time interval was one-month as the final result. To evaluate the intraexaminer reliability, the repeated measurements were examined by the paired t-test (systematic errors) and the Dahlberg formula (casual errors) ${ }^{[13]}$. No significant systematic errors were found $(P>0.1)$, 
and the random errors were small, showing high rates of reproducibility. The normality of distribution of the variables was assessed by Shapiro-Wilks test. Interphase changes (T1-T0) were calculated, and if normally distributed, these were compared using paired t-tests; if this was not the case, the Wilcoxon test was used. Pearson's Chi-square test was applied to determine whether different ways of teeth movement affected the measurement results before and after treatment. Regression analysis was performed to determine the relationship between changes in the alveolar bone area and apex displacement as well as changes in the inclination of incisors. All statistical analyses were performed with GraphPad Prism 8.0.1 and SPSS Statistics with a significance level of 0.05 .

\section{Results}

\section{Incisor tooth movement}

Inclination of incisors related to $\mathrm{FH}$ plane and anatomic position of apex foramen to palatal cortical plate was determined to reflect the 3-dimensional position of the incisors. Although we selected cases with a crowding less than $4 \mathrm{~mm}$, the inclination of central and lateral incisors demonstrated a rather large discrepancy, a result of crowding and irregularity in the maxillary front region. The inclination of central and lateral was significantly reduced after treatment (Table 2). In addition, the distance of apex of central and lateral incisors to palatal cortical plate was significantly reduced with an average of $1.0 \mathrm{~mm}$ and 1.9 respectively, showing that root apex was moved towards the palatal cortical plate (Table 2).

Table 2

Changes in the root position during orthodontic tooth movement (Mean \pm SD).

\begin{tabular}{|c|c|c|c|c|c|c|}
\hline & \multicolumn{3}{|c|}{ Inclination to FH plane $\left(^{(}\right)$} & \multicolumn{3}{|c|}{$\begin{array}{l}\text { Distance from apex to } \\
\text { palatal cortical plate (mm) }\end{array}$} \\
\hline & T0 & $\mathrm{T} 1$ & T1- T0 & T0 & T1 & T1- T0 \\
\hline Central & $119.4 \pm 7.7$ & $111.9 \pm 9.2$ & $-7.5 \pm 10.6^{\star}$ & $6.4 \pm 1.8$ & $5.4 \pm 3.0$ & $-1.0 \pm 2.2^{*}$ \\
\hline Lateral & $117.0 \pm 7.7$ & $113.3 \pm 7.3$ & $-3.7 \pm 9.9 *$ & $5.8 \pm 1.7$ & $3.9 \pm 2.2$ & $-1.9 \pm 2.2^{\star}$ \\
\hline
\end{tabular}

\section{Alveolar bone thickness}

We next explored changes of the buccal, palatal and total ABT at the crestal, mid-root and apical third. The labial ABT of both central and lateral incisors at the mid-root third was increased significantly $(P<$ $0.05)$, while no significant difference was observed at the crestal and apical third. In contrast, the palatal ABT of the central and lateral incisors at all three levels were consistently decreased $(P<0.05)$. Regarding changes in the total $A B T$, significant reduction was observed in both central and lateral incisors at the crestal level, and in lateral incisors at the mid-root third level as well as apical third level $(P<0.05)$ (Table 3). 
Table 3

Changes in alveolar bone thickness at the crestal, mid-root and apical third (Mean \pm SD).

\begin{tabular}{|c|c|c|c|c|}
\hline \multicolumn{5}{|c|}{ Alveolar Bone Thickness (mm) } \\
\hline Tooth & Location & T0 & T1 & P Value \\
\hline \multirow[t]{9}{*}{ Central } & Crestal-labial & $0.8 \pm 0.3$ & $0.8 \pm 0.3$ & $0.2^{\top}$ \\
\hline & Mid-root-labial & $0.7 \pm 0.2$ & $0.9 \pm 0.4$ & $\star \star W$ \\
\hline & Apical-labial & $0.9 \pm 0.3$ & $1.0 \pm 0.6$ & $0.3^{\mathrm{W}}$ \\
\hline & Crestal-palatal & $1.6 \pm 0.4$ & $0.7 \pm 0.9$ & $\star * \star W$ \\
\hline & Mid-root-palatal & $2.9 \pm 0.8$ & $2.2 \pm 1.4$ & $\star \star \star T$ \\
\hline & Apical-palatal & $4.5 \pm 1.4$ & $4.1 \pm 1.8$ & $\star W$ \\
\hline & Crestal-total & $8.3 \pm 0.8$ & $7.8 \pm 1.0$ & $* \star * T$ \\
\hline & Mid-root- total & $8.6 \pm 0.9$ & $8.5 \pm 1.5$ & $0.6^{\top}$ \\
\hline & Apical- total & $8.8 \pm 1.1$ & $9.0 \pm 1.7$ & $0.4^{\mathrm{W}}$ \\
\hline \multirow[t]{9}{*}{ Lateral } & Crestal-labial & $0.7 \pm 0.5$ & $0.6 \pm 0.4$ & $0.3^{\mathrm{W}}$ \\
\hline & Mid-root-labial & $0.5 \pm 0.3$ & $0.6 \pm 0.4$ & $\star * * * T$ \\
\hline & Apical-labial & $0.5 \pm 0.5$ & $0.7 \pm 0.5$ & $0.1^{\mathrm{W}}$ \\
\hline & Crestal-palatal & $1.4 \pm 0.5$ & $0.4 \pm 0.6$ & $\star \star * * T$ \\
\hline & Mid-root-palatal & $2.5 \pm 0.9$ & $1.5 \pm 1.1$ & $\star \star \star W$ \\
\hline & Apical-palatal & $3.8 \pm 1.3$ & $3.17 \pm 1.6$ & $\star \star \star W \mathrm{~W}$ \\
\hline & Crestal-total & $7.9 \pm 0.8$ & $7.1 \pm 0.9$ & $\star \star * * T$ \\
\hline & Mid-root- total & $8.1 \pm 1.2$ & $7.5 \pm 1.2$ & $\star * * * T$ \\
\hline & Apical- total & $8.2 \pm 1.3$ & $7.7 \pm 1.5$ & $* * T$ \\
\hline
\end{tabular}

\section{Alveolar bone height}

In consistence with the reduced $\mathrm{ABA}$, a significant decrease in the palatal $\mathrm{ABH}$ of both central and lateral incisors was observed between T0 and T1 $(\mathrm{P}<0.001)$, indicating palatal alveolar bone resorption. 
However, in terms of labial ABH, no significant difference was observed (Table 4).

Table 4

Changes in the labial and palatal alveolar bone height in central incisors and lateral incisors before (T0) and After treatment (T1).

\begin{tabular}{|c|c|c|c|c|}
\hline \multicolumn{5}{|c|}{ Alveolar Bone Height (mm) (Mean \pm SD) } \\
\hline Tooth & Location & T0 & $\mathrm{T} 1$ & $P$ Value \\
\hline \multirow[t]{2}{*}{ Central incisor } & Labial & $1.6 \pm 0.6$ & $1.6 \pm 0.8$ & $0.1^{\mathrm{W}}$ \\
\hline & Palatal & $1.4 \pm 0.5$ & $3.2 \pm 2.0$ & $\star \star \star * \mathrm{~W}$ \\
\hline \multirow[t]{2}{*}{ Lateral incisor } & Labial & $2.0 \pm 0.8$ & $2.0 \pm 1.5$ & $0.9^{W}$ \\
\hline & Palatal & $1.4 \pm 0.6$ & $4.1 \pm 2.2$ & $\star \star \star * W$ \\
\hline
\end{tabular}

\section{Alveolar bone area}

Decreased palatal ABA was observed in the majority of samples ( $74 \%$ in central incisors and $86 \%$ in lateral incisors). In contrast, the labial ABA was decreased in $36 \%$ of central incisors and $40 \%$ of lateral incisors respectively, while it was increased in $55 \%$ of central incisors and $57 \%$ of lateral incisors. An average bone loss of 20.5 and 40.0 percent on the palatal side was observed in the central and lateral incisors respectively. Further statistical paired t-tests revealed that reduction in the palatal ABA of the central and lateral incisors was statistically significant $(P<0.001)$, while no difference was observed in the labial side $(P>0.05)$ (Fig. 2, Table 5).

Table 5

Changes in the labial and palatal alveolar bone area in central and lateral incisors before (T0) and after treatment (T1) (Mean \pm SD).

\begin{tabular}{|c|c|c|c|c|c|c|}
\hline \multicolumn{7}{|c|}{ Alveolar Bone Area $\left(\mathrm{mm}^{2}\right)$} \\
\hline Tooth & Location & TO & $\mathrm{T} 1$ & T1-T0 & (T1-T0)/T1区\%区 & $P$ Value \\
\hline \multirow[t]{2}{*}{ Central } & labial & $6.2 \pm 2.3$ & $6.1 \pm 2.3$ & $-0.1 \pm 3.1$ & $11.9 \pm 78.1$ & $0.9 \mathrm{~W}$ \\
\hline & palatal & $20.4 \pm 5.4$ & $16.2 \pm 11.1$ & $4.3 \pm 10.5$ & $-20.5 \pm 48.8$ & $\star * \star W$ \\
\hline \multirow[t]{2}{*}{ Lateral } & labial & $4.0 \pm 2.2$ & $4.6 \pm 2.2$ & $0.5 \pm 3.0$ & $29.1 \pm 80.6$ & $0.1^{\mathrm{W}}$ \\
\hline & palatal & $18.2 \pm 6.0$ & $10.6 \pm 7.5$ & $-7.6 \pm 7.7$ & $-40.0 \pm 45.8$ & $\star \star \star W W$ \\
\hline
\end{tabular}




\section{Relationship between changes of labial ABA and tooth movement types}

Among all 152 teeth, the apex of $38(25 \%)$ teeth was moved toward labial side, while the remaining 114 (75\%) was toward palatal side. In the meantime, the inclination of $26(17.1 \%)$ teeth increased, whereas the remaining $126(82.9 \%)$ decreased.

Four different ways of tooth movement were achieved through permutation and combination theory: labial apex displacement $(+)$ \& inclination increase $(+)$, labial apex displacement $(+)$ \& inclination decrease (-), palatal apex displacement (-) \& inclination increase (+), and palatal apex displacement (-) \& inclination decrease (-). Among them, there was no labial apex displacement (+) \& inclination increase (+) in the subjects. Pearson's Chi-square test was applied to determine whether three ways of tooth movement affected the labial ABA before and after treatment, which showed a strong correlation between labial ABA changes and tooth movement type $(P<0.001)$ (Table 6). Labially-displaced apex was correlated with decreased labial $A B A$, while palatally-displaced apex was correlated with increased labial ABA.

\section{Table 6}

Frequency of labial alveolar bone area (ABA) increase and decrease in upper four incisors among different ways of tooth movement using a Pearson $X^{2}$ Test $(n=152)$.

\begin{tabular}{|c|c|c|c|c|c|}
\hline Apex displacement & Inclination & $\begin{array}{l}\text { Labial ABA } \\
\text { Increase (+) }\end{array}$ & $\begin{array}{l}\text { Labial ABA } \\
\text { Decrease (-) }\end{array}$ & $x^{2}$ & P Value \\
\hline Labial (+) & Decrease $(-)$ & 9 & 29 & \multirow[t]{3}{*}{33.1} & \multirow[t]{3}{*}{$\star \star \star ~$} \\
\hline Palatal (-) & Increase (+) & 23 & 3 & & \\
\hline Palatal (-) & Decrease $(-)$ & 61 & 27 & & \\
\hline$\star \star \star, P<0.001$. & & & & & \\
\hline
\end{tabular}

As a reduction in palatal $A B A$ was observed in most patients, a multivariate linear regression analysis was used to further explore the relationship between alterations of palatal ABA and tooth movement type, showing that the changes (T1-T0) of palatal ABA were remarkably correlated with the apex displacement and changes of inclination (T1-T0) before and after treatment $(F=107.939, P<0.001)$ ( Table 7). This model can be also described by using this equation: Changes of palatal ABA (T1-T0) $=-3.258-0.139 \times$ changes of inclination (T1-T0) $+2.533 \times$ apex displacement (T1-T0) (Table 7). 
Table 7

Multivariate linear regression analysis of apex displacement and changes in inclination (T1-T0) for the changes of palatal alveolar bone area (ABA) (T1-T0) in maxillary incisors.

\begin{tabular}{|c|c|c|c|c|c|}
\hline dependent variable & $\begin{array}{l}\text { constant and } \\
\text { influencing factors }\end{array}$ & $\mathrm{R}^{2}$ & F value & b (SE) & $P$ value \\
\hline \multirow{3}{*}{$\begin{array}{l}\text { Changes in palatal } \\
\text { ABA(T1-T0) }\end{array}$} & Constant & \multirow[t]{3}{*}{0.6} & \multirow[t]{3}{*}{$108.0 * \star \star$} & $-3.3(0.8)$ & $\star \star \star *$ \\
\hline & Changes in Inclination(T1-T0) & & & $-0.1(0.1)$ & * \\
\hline & Apex Displacement & & & $2.5(0.3)$ & $\star \star \star *$ \\
\hline
\end{tabular}

The equation can be used to predict changes of palatal ABA with certain values of the predictors. For example, the predicted changes of palatal ABA for a patient with palatal apex displacement of $-3 \mathrm{~mm}$ and decrease of $8^{\circ}$ in inclination is $-3.258-0.139 \times(-8)+2.533 \times(-3)$, which equals to $-7.521 \mathrm{~mm}^{2}$.

\section{Discussion}

Dentists have been seeking personalized approaches to improve treatment outcome and avoid deleterious sequelae for a long time ${ }^{[15]}$. The new era of precision medicine requires a personalized, or individualized treatment plan and case management ${ }^{[16]}$. The use of digital orthodontic setups has grown quickly to aid individualized plan, mimic tooth movement and predict treatment outcome ${ }^{[17]}$. Periodontal health is of great concern for the orthodontists and periodontists, especially in the inter-disciplinary management of bimaxillary protrusion patients. Three-dimensional CBCT imaging have exponentially enhanced the capability to evaluate regional anatomy in the alveolar process and assess periodontal risks during tooth movement ${ }^{[7]}$. The principal finding of the present research was that apex displacement is the major factor that contributed to decreased palatal $A B A$ and the equation that integrates apex displacement and incisor inclination may help periodontists and orthodontists evaluate potential risks for deleterious sequelae of periodontal bone resorption.

Alveolar bone, originates from the dental follicle during embryogenesis, is unique in its dynamic remodeling capacity during tooth eruption and $\mathrm{OTM}^{[7]}$. It has been long accepted that OTM is a dynamic process whereby the application of orthodontic force induces bone resorption on the pressure side and bone apposition on the tension side, which maintains structural integrity of the alveolar bone ${ }^{[6]}$. Although the alveolar bone might be dynamically remodeled to house moving teeth in growing adolescents, OTM is limited by the cortical plates of the alveolus at the level of the incisor apices, which can be regarded as "orthodontic walls", in non-growing adults ${ }^{[4]}$. Moving teeth out of these boundaries may lead to occurrence of severe iatrogenic sequelae of alveolar bone resorption, and this is especially disastrous in adult patients. The width of the anterior palate at the level of the apex remained unaltered despite long- 
term incisor retraction in adult patients ${ }^{[18]}$. Indeed, we observed significant decrease in the height, thickness and area of alveolar bone on the palatal side.

The acumen of CBCT may help orthodontists and periodontists assess periodontal bone status and remodeling in OTM. Stages of alignment, bite opening and space closing are integrated processes in extraction cases. It has been demonstrated that alignment of anterior teeth by tipping leaded to significant vertical and horizontal loss of alveolar bone in non-extraction cases ${ }^{[19-21]}$. In addition, Yodthong et. al. investigated the effect of incisor retraction on changes of alveolar bone, CBCT was taken before retraction and after 6 month of retraction, and they reported an increase of $0.4 \mathrm{~mm}$ in ABT at the crestal level on the labial side, $0.2 \mathrm{~mm}$ and $0.6 \mathrm{~mm}$ decrease at crestal and apical level on the palatal side respectively for the central incisors, indicating that incisor retraction is a risk of alveolar bone resorption in bimaxillary protrusion patients; however, the total thickness of the alveolar bone even increased an average of $0.6 \mathrm{~mm}$ at the apical level ${ }^{[22]}$. Sarikaya et. al. reported that no changes in the labial side were observed, while decrease in ABT in the maxillary arch was observed in four premolar extraction cases with $0.7 \mathrm{~mm}$ and $1.2 \mathrm{~mm}$ at the crestal and mid-root level ${ }^{[3]}$. Our results were consistent with Ahn et. al. that remarkable bone absorption can be found in all three levels on the palatal side, while bone thickness on the labial side increased in the middle third, by $0.27 \mathrm{~mm}$ for upper central incisors and by $0.65 \mathrm{~mm}$ for lateral incisors, with statistically significant differences ${ }^{[23]}$.

In the process of assessing the periodontal risk, dentists should not only evaluate anatomic features of alveolar bone before treatment, but also the remodeling potential and 3-dimensional position of targeted teeth in the alveolus ${ }^{[24]}$. 3D imaging techniques may help orthodontic-periodontic interdisciplinary coordination in managing periodontal iatrogenic effects ${ }^{[25]}$. Consistent absorption of alveolar bone on the palatal side in our study and in Ahn et. al. ${ }^{[23]}$ indicates that retraction actually moves incisors "through-the-bone." Despite the increase in alveolar bone thickness and area on the buccal side, decrease in the total thickness and area in the incisor region suggests that orthodontic treatment may lead to increased risks for periodontal sequelae.

Digital setups have been widely used for diagnosis, treatment planning, indirect bonding, simulating treatment, and designing and producing orthodontic appliances, especially in the aligner techniques ${ }^{[17,26 \text {, }}$ 27]. Much emphasis has been laid on crown position rather than root position because spatial position of roots is not available until the emergence of CBCT imaging and roots are usually not directly related to esthetics and occlusal contact ${ }^{[28]}$. However, if the alveolar bone is not taken into consideration, such digital setup may push the tooth out of the bone for considerable distance.

Both the height and thickness of alveolar bone are critical factors to protect the teeth from plaqueinduced (i.e., periodontitis) and non-plaque-induced gingival lesions ${ }^{[29]}$. Thinner alveolar wall after incisor retraction may lead to dehiscences or fenestrations that compromise alveolar bone support ${ }^{[14]}$. In order to investigate overall effect of incisor retraction on alveolar remodeling, we made a preliminary attempt to describe changes in alveolar bone area, which reflected changes in $A B L$ and $A B T$; we found that apical 
displacement and alterations in inclination were significantly related to ABA on the palatal side; moreover, we developed an equation to describe changes in $A B A$, which described that changes of palatal $A B A$ (T1T0) is equal to $(-3.258-0.139 \times$ changes of inclination (T1-T0) $+2.533 \times$ apex displacement (T1-T0)).

We utilized changes in the inclination of each individual incisor rather than measurements from cephalometric to generate an equation for prediction of individual palatal ABA. Such equation may customize both periodontal and orthodontic consideration in adult patients, especially determining whether bone augmentation surgery was needed to aid orthodontic therapy, commonly recognized as surgically facilitated orthodontic therapy [SFOT] ${ }^{[30]}$ or periodontally accelerated osteogenic orthodontics [PAOO] $)^{[31]}$. Our present equation further supports the conception that bodily retraction would lead to proximity of root apex to palatal cortical plate and extensive alveolar bone resorption ${ }^{[32]}$, and this jeopardy is particularly pertinent in adults as numbers and regeneration capability of osteoblasts in the periosteum reduces with aging ${ }^{[33]}$.

Many factors may influence OTM and alveolar bone remodeling in the periodontium, such as force magnitude (light or heavy), force type (intermittent, interrupted or continuous), age( adolescents or adults), distance of movement, type of OTM(tipping, controlled tipping, translation, intrusion, extrusion), speed of space closure ${ }^{[22]}$. For example, intrusion of incisors into a wider alveolus may increase alveolar bone support, thereby compensating the palatal bone resorption [34]; however, excessive intrusion may lead to remarkable root resorption ${ }^{[35]}$. Cortical plates at the root apex level did not remodel in adult patients ${ }^{[4]}$; therefore, it may be utilized as a reliable mark to reflect the position of incisors. In our present study, we did not measure the distance of retraction for individual teeth, because the distance of root apex to palatal plates and inclination of incisors actually reflected changes in the position of incisors. Another issue was that we did not include the vertical position of root apex. Only minimal intrusion of incisors can be observed during retraction of incisors in bimaxillary protrusion patients ${ }^{[22]}$, and large quantities of incisor intrusion can only be observed in class II patients, especially division 2 type with reverse curve of Spe ${ }^{[36]}$. Data from the 2-dimensional changes in the lateral cephalograms were most often utilized to represent overall intrusion ${ }^{[22,36,37]}$; therefor, we did not further investigate influence of intrusion of incisor in 2-dimensional cephalometric on ABA.

We utilized adult patients to minimize the influence of growth on alveolar bone remodeling; therefore, interpretation of data in adolescents should be cautious. A key question critical in alveolar bone remodeling during OTM is elasticity and flexibility of alveolar bone, which undergoes rapid remodeling with low mineralization and stiffness even in adults ${ }^{[38]}$. More CBCT data, especially from growing subjects, are needed to better predict changes in the periodontium and optimize periodontal risk assessment. Although CBCT may reduce periodontal risks in OTM, ALARA (As Low As Reasonably Achievable) principle should be adhered to, especially in growing adolescents. Each subject should be evaluated individually based on their unique treatment needs and set of circumstances ${ }^{[7]}$. Digital set-up provides dentists unprecedented opportunities to predict the final position of the crowns before the 
treatment ${ }^{[39]}$. Our present research indicated that with the help of CBCT imaging, dentists may further determine the final root position in the alveolus in the future.

\section{Conclusions}

1. Retraction of incisors in bimaxillary protrusion patients leaded to significant bone resorption on the palatal side, which may compromise periodontal bone support;

2. Palatal displacement of root apex was highly correlated with palatal bone resorption;

3. The area of bone resorption might be predicted by an equation that incorporated the displacement of root apex and changes in the incisor inclination.

\section{Abbreviations}

CBCT: Cone-beam computed tomography; ABT: Alveolar bone thickness: ABH: alveolar bone height; $A B A$ : alveolar bone area; OTM: orthodontic tooth movement; SWA: straight wire appliances; TADs: temporary anchorage devices; CEJ: cementoenamel junction; FH plane: Frankfurt horizontal plane.

\section{Declarations}

\section{Acknowledgements}

The authors are grateful to the colleagues, especially Dr. Jun Ji, at the Nanjing Stomatological Hospital, Medical School of Nanjing University for the support of present study.

\section{Authors' Contributions}

$\mathrm{HL}$ and $\mathrm{LL}$ developed the concept and design of this study. HM conducted data collection and measurement. $\mathrm{HL}$ and LL supervised the data assessment. HM, AY, and YP performed statistical analyses, and all authors contributed to the interpretation of the data. HM, AY, and YP drafted the manuscript. All authors read and approved the final manuscript.

\section{Funding}

This research is supported by Nanjing Medical Science and technique Development Foundation (QRX17081 and QRX17025), National Natural Science Foundation of China (81670996). The funding body supported data analysis and writing the manuscript.

\section{Availability of data and materials}

The raw data in this study are available by contacting corresponding author Dr. Lang Lei, China-Email address: leilangdental@163.com.

\section{Ethics approval and consent to participate}


The study protocol was evaluated and approved by the local ethics committee (Nanjing Stomatological Hospital, Medical School of Nanjing University, No. 2019NL-064(KS)). Written consent was obtained from participants or their parents (for participants less than 16 years old).

\section{Consent for publication}

Not applicable.

\section{Competing interests}

The authors declare that they have no competing interests.

\section{Author details}

Huimin Mao, Andi Yang, Yue Pan \& Lang Lei are from Department of Orthodontics, Nanjing Stomatological Hospital, Medical School of Nanjing University, Nanjing, China; Houxuan Li is from Department of Periodontics, Nanjing Stomatological Hospital, Medical School of Nanjing University, Nanjing, China.

\section{References}

[1] Leonardi R, Annunziata A, Licciardello V, Barbato E. Soft tissue changes following the extraction of premolars in nongrowing patients with bimaxillary protrusion. A systematic review. Angle Orthod. 2010. 80(1): 211-6.

[2] Wang Q, Jia P, Anderson NK, Wang L, Lin J. Changes of pharyngeal airway size and hyoid bone position following orthodontic treatment of Class I bimaxillary protrusion. Angle Orthod. 2012. 82(1): 11521.

[3] Sarikaya S, Haydar B, Ciğer S, Ariyürek M. Changes in alveolar bone thickness due to retraction of anterior teeth. Am J Orthod Dentofacial Orthop. 2002. 122(1): 15-26.

[4] Wainwright WM. Faciolingual tooth movement: its influence on the root and cortical plate. Am J Orthod. 1973. 64(3): 278-302.

[5] Wehrbein H, Fuhrmann RA, Diedrich PR. Periodontal conditions after facial root tipping and palatal root torque of incisors. Am J Orthod Dentofacial Orthop. 1994. 106(5): 455-62.

[6] Meikle MC. The tissue, cellular, and molecular regulation of orthodontic tooth movement: 100 years after Carl Sandstedt. Eur J Orthod. 2006. 28(3): 221-40.

[7] Mandelaris GA, Neiva R, Chambrone L. Cone-Beam Computed Tomography and Interdisciplinary Dentofacial Therapy: An American Academy of Periodontology Best Evidence Review Focusing on Risk 
Assessment of the Dentoalveolar Bone Changes Influenced by Tooth Movement. J Periodontol. 2017. 88(10): 960-977.

[8] Singh S, Jayan B. Comparative Evaluation of Periodontally Accelerated Osteogenic Orthodontics (PAO0) Versus Conventional Orthodontic Tooth Movement in Adult Patients with Bimaxillary Dentoalveolar Protrusion. Int J Periodontics Restorative Dent. 2019. 39(4): 571-577.

[9] Padala S, Tee BC, Beck FM, Elias K, Kim DG, Sun Z. The usefulness of cone-beam computed tomography gray values for alveolar bone linear measurements. Angle Orthod. 2018. 88(2): 227-232.

[10] Damstra J, Fourie Z, Huddleston Slater JJ, Ren Y. Accuracy of linear measurements from conebeam computed tomography-derived surface models of different voxel sizes. Am J Orthod Dentofacial Orthop. 2010. 137(1): 16.e1-6; discussion 16-7.

[11] Tominaga JY, Tanaka M, Koga Y, Gonzales C, Kobayashi M, Yoshida N. Optimal loading conditions for controlled movement of anterior teeth in sliding mechanics. Angle Orthod. 2009. 79(6): 1102-7.

[12] Krieger E, Seiferth J, Saric I, Jung BA, Wehrbein H. Accuracy of Invisalign ${ }^{\circledR}$ treatments in the anterior tooth region. First results. J Orofac Orthop. 2011. 72(2): 141-9.

[13] Kim KW, Kim SJ, Lee JY, et al. Apical root displacement is a critical risk factor for apical root resorption after orthodontic treatment. Angle Orthod. 2018. 88(6): 740-747.

[14] Vardimon AD, Oren E, Ben-Bassat Y. Cortical bone remodeling/tooth movement ratio during maxillary incisor retraction with tip versus torque movements. Am J Orthod Dentofacial Orthop. 1998. 114(5): 520-9.

[15] Bartold PM. Lifestyle and periodontitis: The emergence of personalized periodontics. Periodontol 2000. 2018. 78(1): 7-11.

[16] Flores M, Glusman G, Brogaard K, Price ND, Hood L. P4 medicine: how systems medicine will transform the healthcare sector and society. Per Med. 2013. 10(6): 565-576.

[17] Fabels LN, Nijkamp PG. Interexaminer and intraexaminer reliabilities of 3-dimensional orthodontic digital setups. Am J Orthod Dentofacial Orthop. 2014. 146(6): 806-11.

[18] Handelman CS. The anterior alveolus: its importance in limiting orthodontic treatment and its influence on the occurrence of iatrogenic sequelae. Angle Orthod. 1996. 66(2): 95-109; discussion 109-10.

[19] Garlock DT, Buschang PH, Araujo EA, Behrents RG, Kim KB. Evaluation of marginal alveolar bone in the anterior mandible with pretreatment and posttreatment computed tomography in nonextraction patients. Am J Orthod Dentofacial Orthop. 2016. 149(2): 192-201. 
[20] Lund H, Gröndahl K, Gröndahl HG. Cone beam computed tomography evaluations of marginal alveolar bone before and after orthodontic treatment combined with premolar extractions. Eur J Oral Sci. 2012. 120(3): 201-11.

[21] Morais JF, Melsen B, de Freitas K, Castello Branco N, Garib DG, Cattaneo PM. Evaluation of maxillary buccal alveolar bone before and after orthodontic alignment without extractions: A cone beam computed tomographic study. Angle Orthod. 2018. 88(6): 748-756.

[22] Yodthong N, Charoemratrote C, Leethanakul C. Factors related to alveolar bone thickness during upper incisor retraction. Angle Orthod. 2013. 83(3): 394-401.

[23] Ahn HW, Moon SC, Baek SH. Morphometric evaluation of changes in the alveolar bone and roots of the maxillary anterior teeth before and after en masse retraction using cone-beam computed tomography. Angle Orthod. 2013. 83(2): 212-21.

[24] Lee RJ, Weissheimer A, Pham J, et al. Three-dimensional monitoring of root movement during orthodontic treatment. Am J Orthod Dentofacial Orthop. 2015. 147(1): 132-42.

[25] Hodges RJ, Atchison KA, White SC. Impact of cone-beam computed tomography on orthodontic diagnosis and treatment planning. Am J Orthod Dentofacial Orthop. 2013. 143(5): 665-74.

[26] Grauer D, Proffit WR. Accuracy in tooth positioning with a fully customized lingual orthodontic appliance. Am J Orthod Dentofacial Orthop. 2011. 140(3): 433-43.

[27] Im J, Cha JY, Lee KJ, Yu HS, Hwang CJ. Comparison of virtual and manual tooth setups with digital and plaster models in extraction cases. Am J Orthod Dentofacial Orthop. 2014. 145(4): 434-42.

[28] Germane N, Bentley BE, Isaacson RJ. Three biologic variables modifying faciolingual tooth angulation by straight-wire appliances. Am J Orthod Dentofacial Orthop. 1989. 96(4): 312-9.

[29] Chan HL, Garaicoa-Pazmino C, Suarez F, et al. Incidence of implant buccal plate fenestration in the esthetic zone: a cone beam computed tomography study. Int J Oral Maxillofac Implants. 2014. 29(1): 171-7.

[30] Roblee RD, Bolding SL, Landers JM. Surgically facilitated orthodontic therapy: a new tool for optimal interdisciplinary results. Compend Contin Educ Dent. 2009. 30(5): 264-75; quiz 276, 278.

[31] Wilcko WM, Wilcko T, Bouquot JE, Ferguson DJ. Rapid orthodontics with alveolar reshaping: two case reports of decrowding. Int J Periodontics Restorative Dent. 2001. 21(1): 9-19.

[32] Meikle MC. The dentomaxillary complex and overjet correction in Class II, division 1 malocclusion: objectives of skeletal and alveolar remodeling. Am J Orthod. 1980. 77(2): 184-97. 
[33] Ferretti C, Lucarini G, Andreoni C, et al. Human Periosteal Derived Stem Cell Potential: The Impact of age. Stem Cell Rev. 2015. 11(3): 487-500.

[34] Deng $Y$, Sun $Y, X u T$. Evaluation of root resorption after comprehensive orthodontic treatment using cone beam computed tomography (CBCT): a meta-analysis. BMC Oral Health. 2018. 18(1): 116.

[35] de Almeida MR, Marçal A, Fernandes T, Vasconcelos JB, de Almeida RR, Nanda R. A comparative study of the effect of the intrusion arch and straight wire mechanics on incisor root resorption: $A$ randomized, controlled trial. Angle Orthod. 2018. 88(1): 20-26.

[36] Kale Varlık S, Onur AÖ, Türköz Ç. Deepbite correction with incisor intrusion in adults: a long-term cephalometric study. Am J Orthod Dentofacial Orthop. 2013. 144(3): 414-9.

[37] Ng J, Major PW, Heo G, Flores-Mir C. True incisor intrusion attained during orthodontic treatment: a systematic review and meta-analysis. Am J Orthod Dentofacial Orthop. 2005. 128(2): 212-9.

[38] Cho MI, Garant PR. Development and general structure of the periodontium. Periodontol 2000. 2000. 24: 9-27.

[39] Staderini E, Guglielmi F, Cornelis MA, Cattaneo PM. Three-dimensional prediction of roots position through cone-beam computed tomography scans-digital model superimposition: A novel method. Orthod Craniofac Res. 2019. 22(1): 16-23.

\section{Figures}


A.

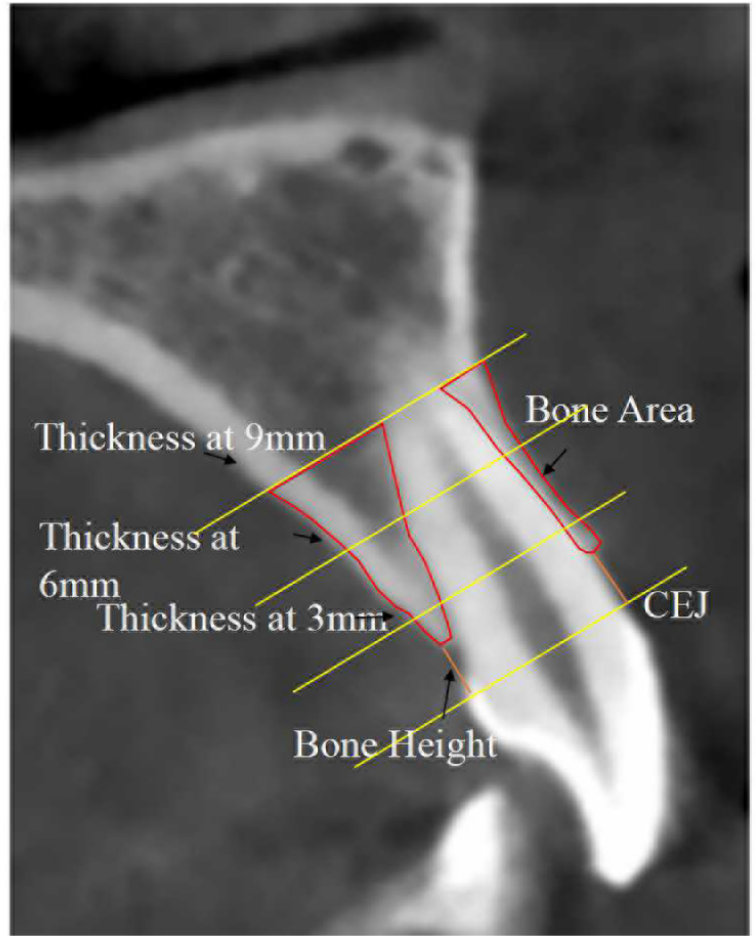

B.

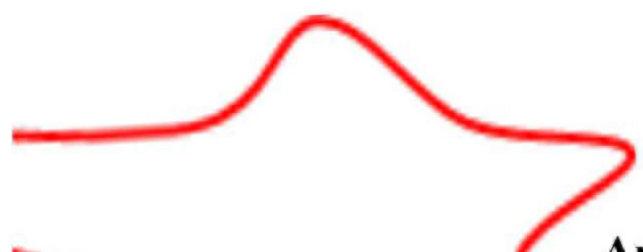

Apical level

Mid-root level

Crestal level

CEJ

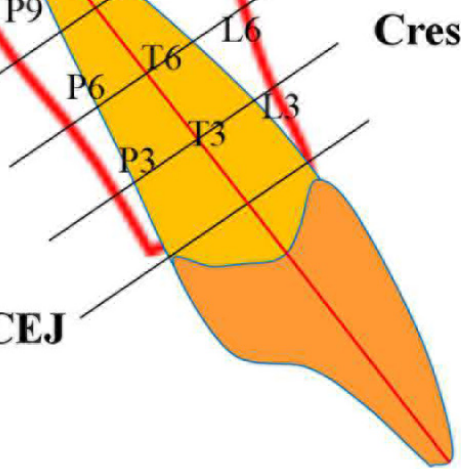

\section{Figure 1}

Determination of alveolar bone morphology in incisors. Alveolar bone thickness was measured at the apical, mid-root and crestal level. CEJ: cementoenamel junction. 
A.

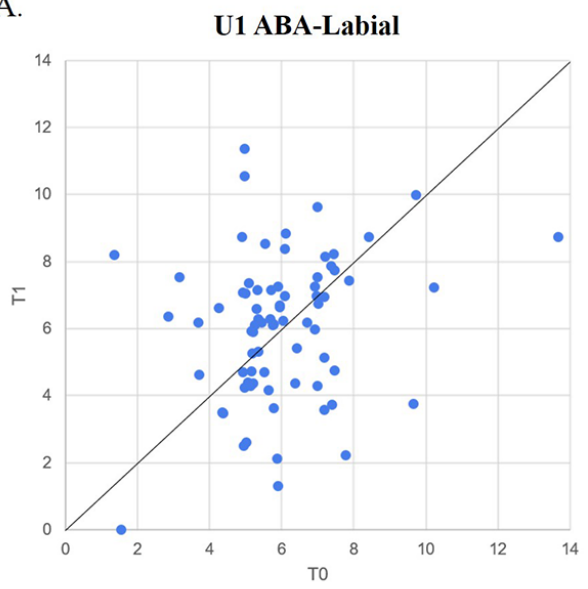

$\mathrm{N}$ under line: $27(36 \%)$; on line: $7(9 \%)$; above line: $42(55 \%)$

C.

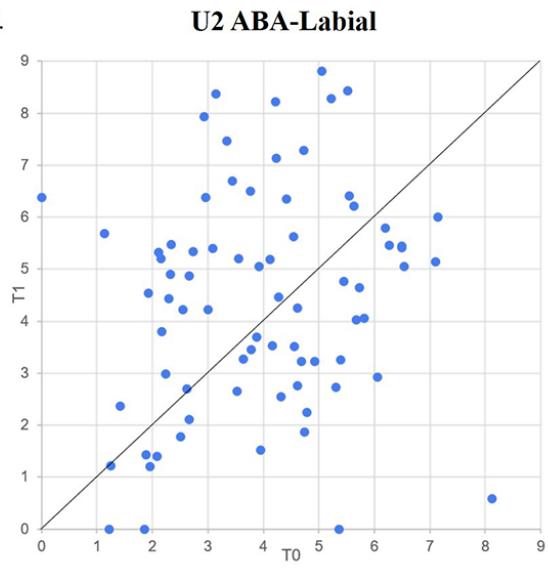

$\mathrm{N}$ under line: $31(40 \%)$; on line: $2(3 \%)$; above line: $43(57 \%)$
B.

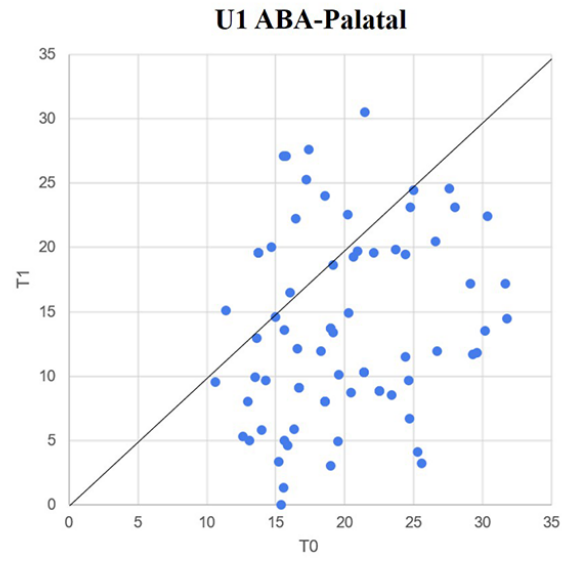

N Under line: 60(79\%); on line: 4(5\%); above line: $16(10 \%)$

D.

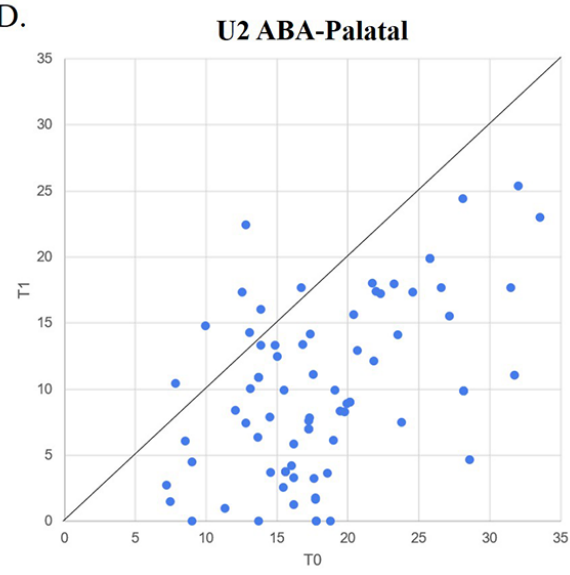

N Under line: 69(91\%); on line:0 (0\%); above line: $7(9 \%)$

\section{Figure 2}

Scatterplots showing the changes in alveolar bone area at T0 and T1. 

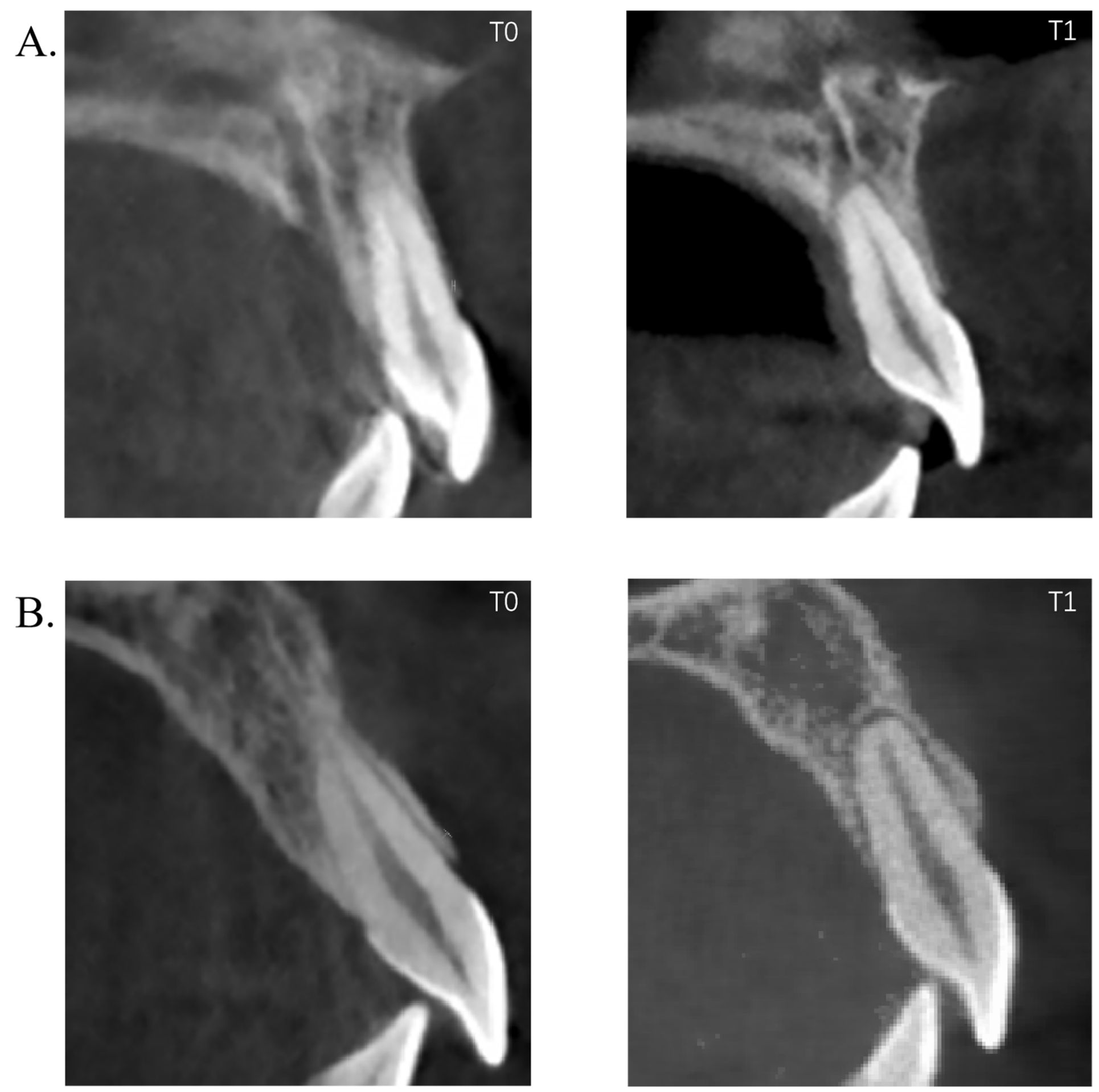

\section{Figure 3}

Characteristic Changes in the alveolar bone during incisor retraction. A. Typical alteration in the alveolar bone at the central incisor with a massive palatal apex displacement, i.e. bodily movement; B. Typical alveolar bone remodeling at the central incisor with a large change in the inclination, i.e. tipping. 